\title{
Pd-nanoparticles as efficient catalyst for Suzuki and Stille coupling reactions of aryl halides in ionic liquids
}

\author{
Vincenzo Calò, Angelo Nacci,* Antonio Monopoli and Floriana Montingelli \\ SUPPORTING INFORMATION
}

\section{Table of Contents}

General Methods S2

Peak assignements of the reaction products. S2

${ }^{1} \mathrm{H}-\mathrm{NMR}$ spectra of the reaction products S3-S9 
General Methods. All chemicals used for synthetic procedures, including the tetraalkylammonium salts and the $1,5 \mathrm{M}$ aqueosus solution of $\mathrm{Bu}_{4} \mathrm{NOH}$ were commercially available. Solutions were concentrated in vacuo with a rotary evaporator, and the residue was purified using a short pad of silica gel unless specified otherwise.

The reaction products have been identified by comparison of their spectral data with those reported in the literature. ${ }^{1} \mathrm{H}$ NMR spectra follow:

Biphenyl: ${ }^{1} \mathrm{H}$ NMR (CDCl $\left.3,500 \mathrm{MHz}\right)$ 7.36-7.42 (m, 2H), 7.45-7.51 (m, 4H), 7.62-7.68 (m, 4H).

4-Methyl-biphenyl: ${ }^{1} \mathrm{H}$ NMR $\left(\mathrm{CDCl}_{3}, 500 \mathrm{MHz}\right) 2.43$ (s, 3H), 7.27-7.31 (m, 2H), 7.34-7.39 (m, 1H), 7.43-7.49 (m, 2H), 7.52-7.5 (m, 2H), 7.60-7.64 (m, 2H).

4-Nitro-biphenyl: ${ }^{1} \mathrm{H}$ NMR $\left(\mathrm{CDCl}_{3}, 500 \mathrm{MHz}\right)$ 7.42-7.54 (m, 3H), 7.60-7.65 (m, 2H), 7.70-7.76 (m, $2 \mathrm{H}), 8.26-8.32(\mathrm{~m}, 2 \mathrm{H})$.

4-Methoxy-biphenyl: ${ }^{1} \mathrm{H}$ NMR $\left(\mathrm{CDCl}_{3}, 500 \mathrm{MHz}\right) 3.86$ (s, 3H), 6.96-7.00 (m, 2H), 7.27-7.34 (m, 1H), 7.39-7.45 (m, 2H), 7.51-7.58 (m, 4H).

1-Biphenyl-4-yl-ethanone: ${ }^{1} \mathrm{H}$ NMR ( $\left.\mathrm{CDCl}_{3}, 500 \mathrm{MHz}\right) 2.64$ (s, 3H), 7.37-7.43 (m, 1H), 7.45-7.50 (m, 2H), 7.60-7.65 (m, 2H), 7.67-7.71 (m, 2H), 8.01-8.08 (m, 2H).

4'-Methoxy-4-nitro-biphenyl: ${ }^{1} \mathrm{H}$ NMR $\left(\mathrm{CDCl}_{3}, 500 \mathrm{MHz}\right) 3.87$ (s, 3H), 6.99-7.03 (m, 2H), 7.557.59 (m, 2H), 7.65-7.69 (m, 2H), 8.23-8.26 (m, 2H).

1-(4'-Methoxy-biphenyl-4-yl)-ethanone: ${ }^{1} \mathrm{H}$ NMR $\left(\mathrm{CDCl}_{3}, 500 \mathrm{MHz}\right) 2.62$ (s, 3H), 3.86 (s, 3H), 6.98-7.02 (m, 2H), 7.56-7.60 (m, 2H), 7.62-7.65 (m, 2H), 7.98-8.02 (m, 2H). 


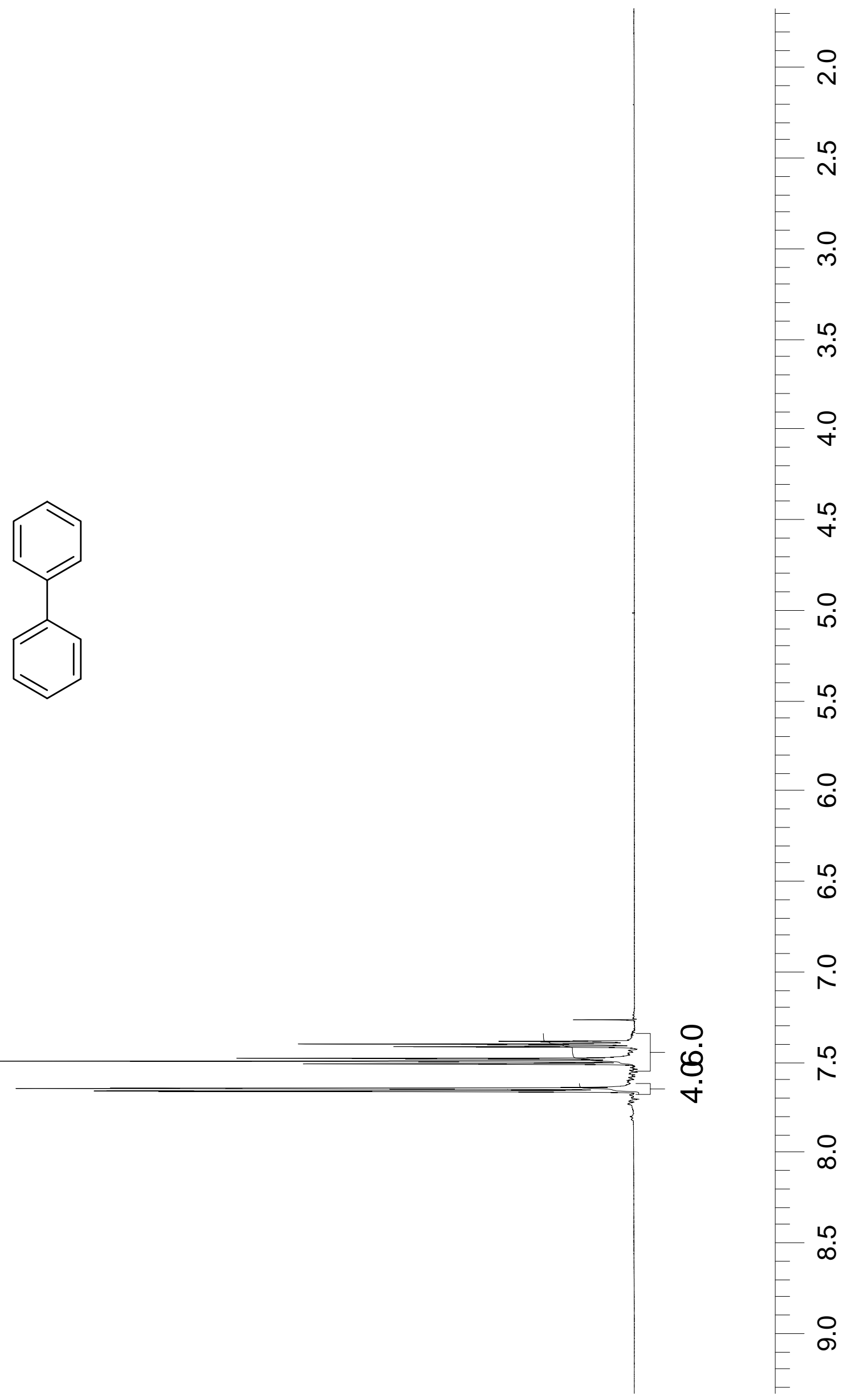




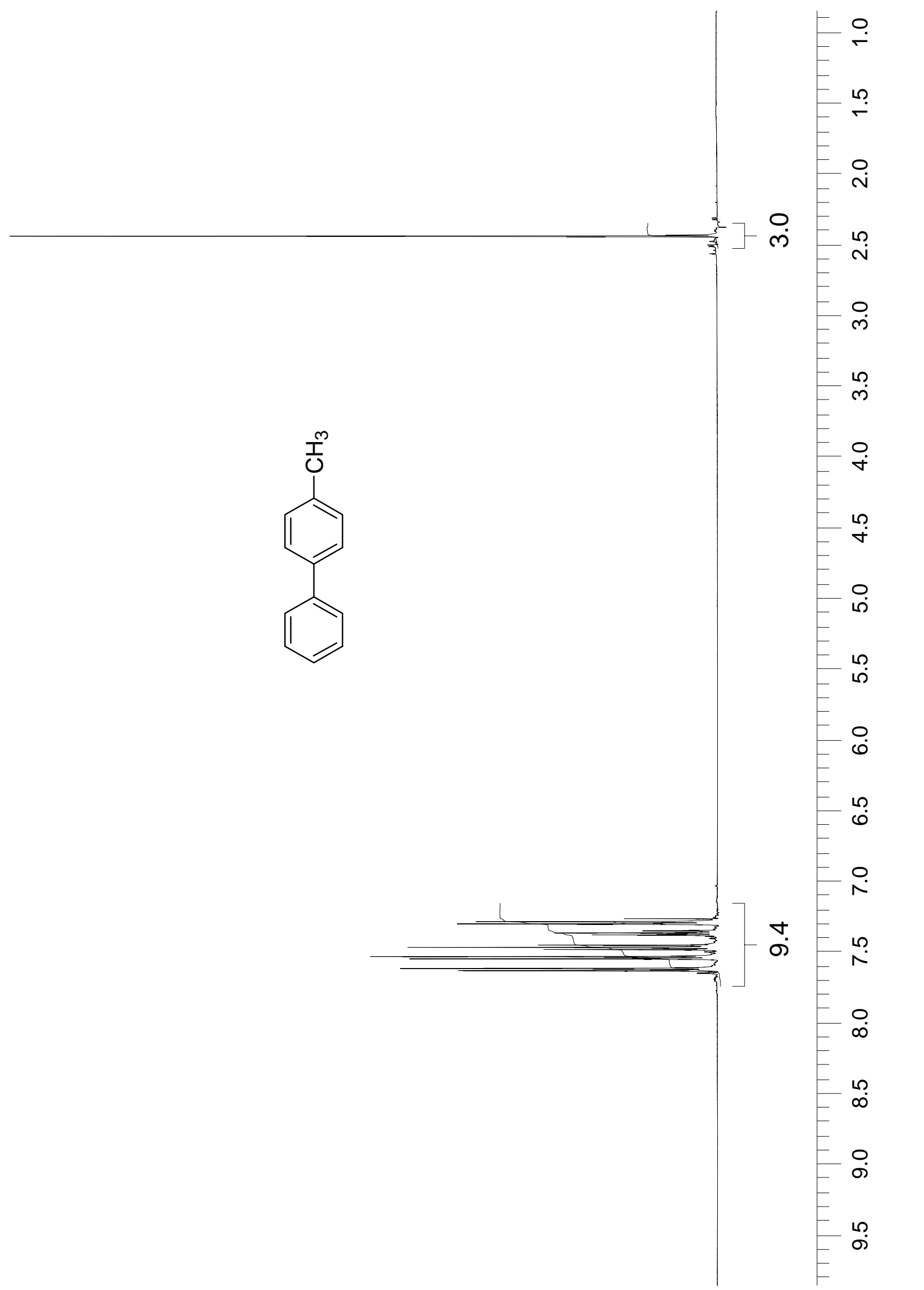




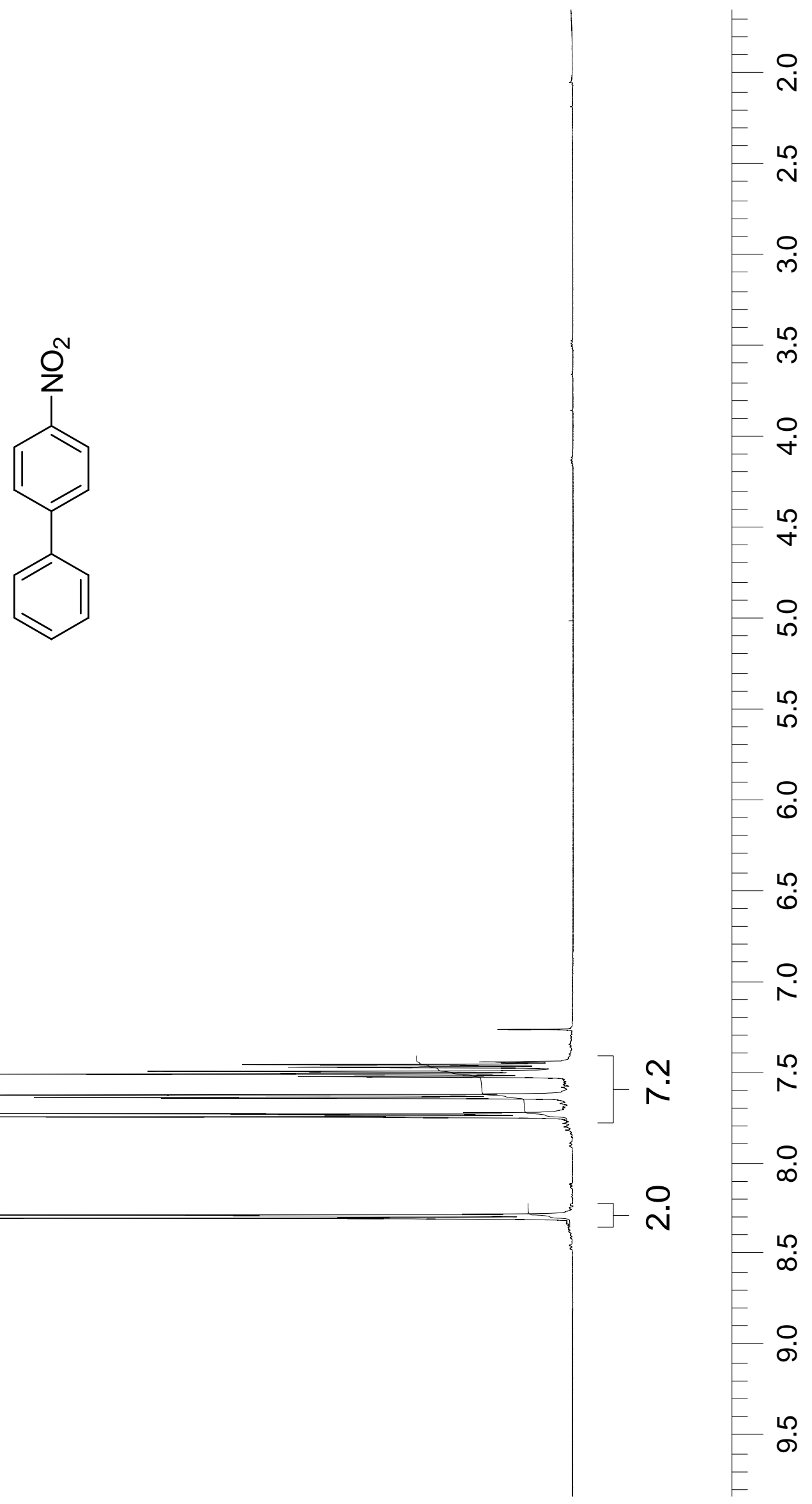




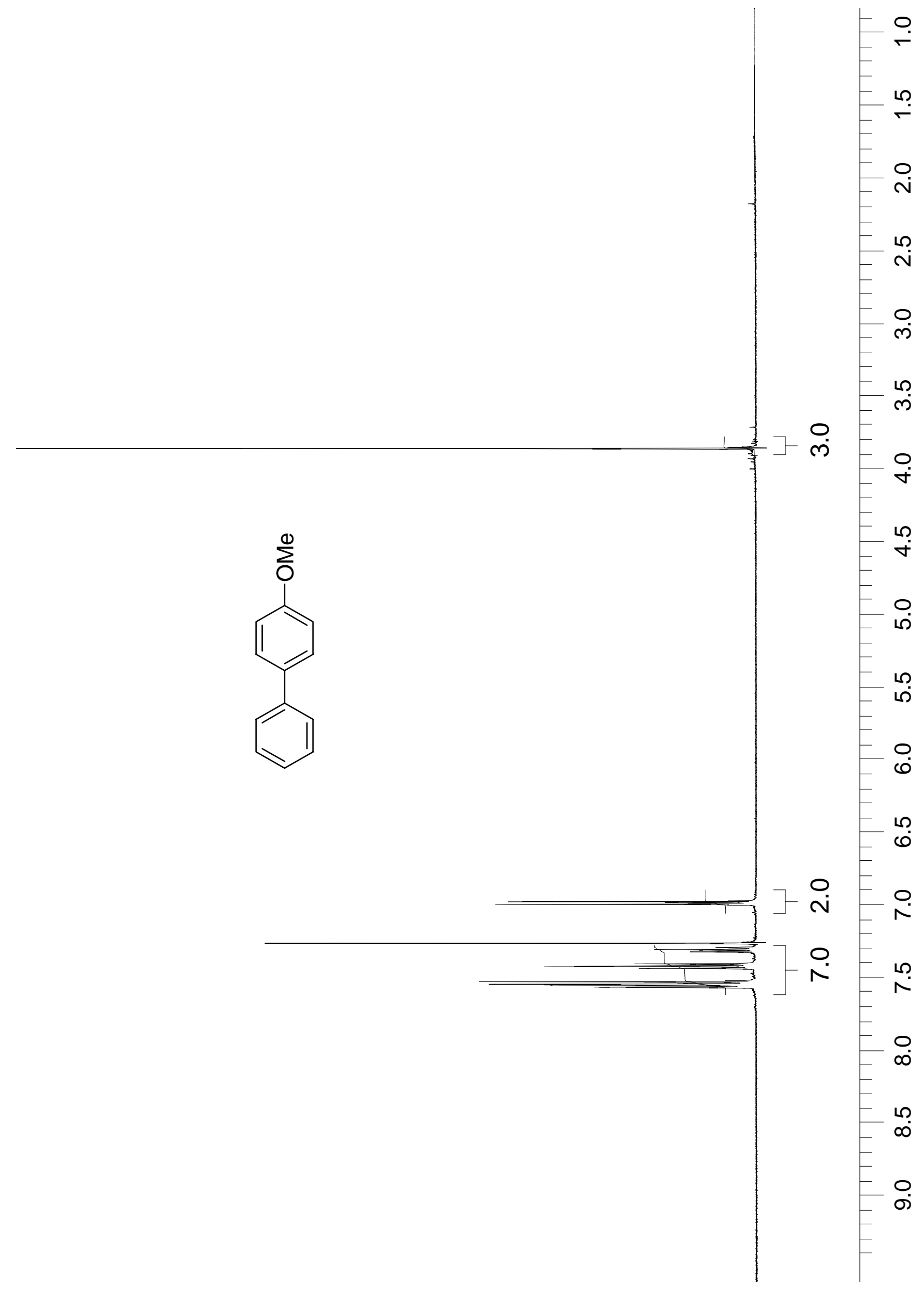




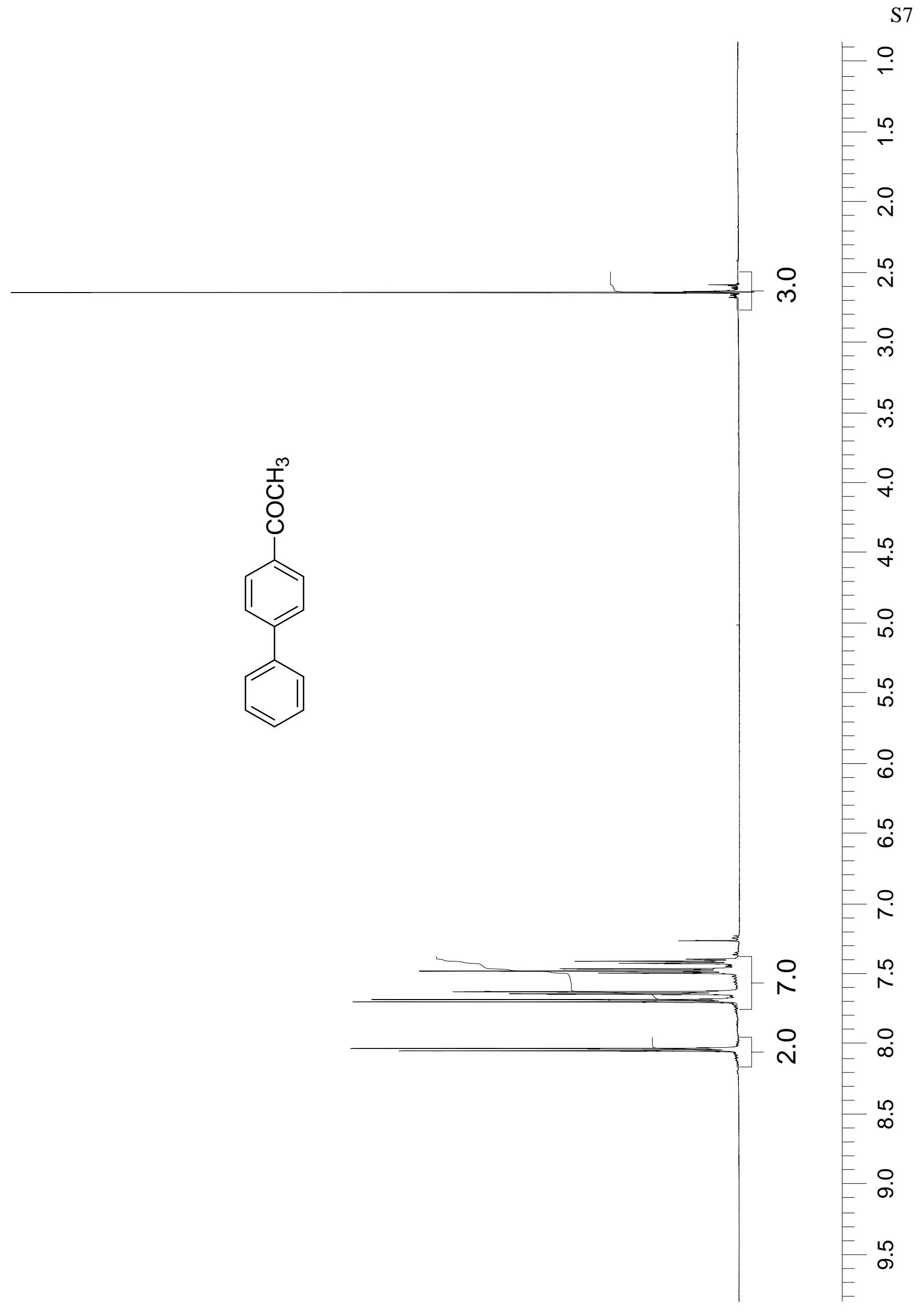




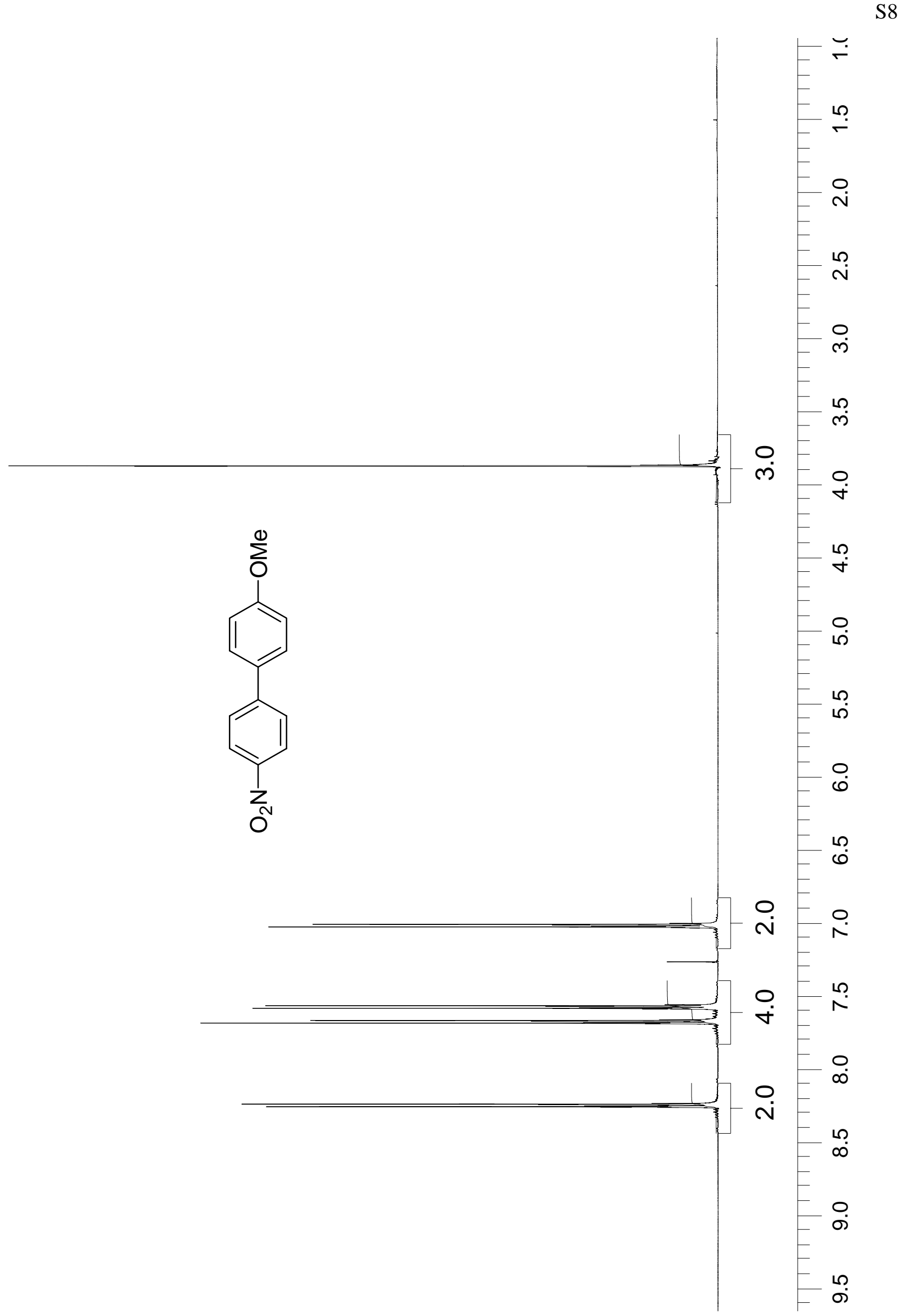




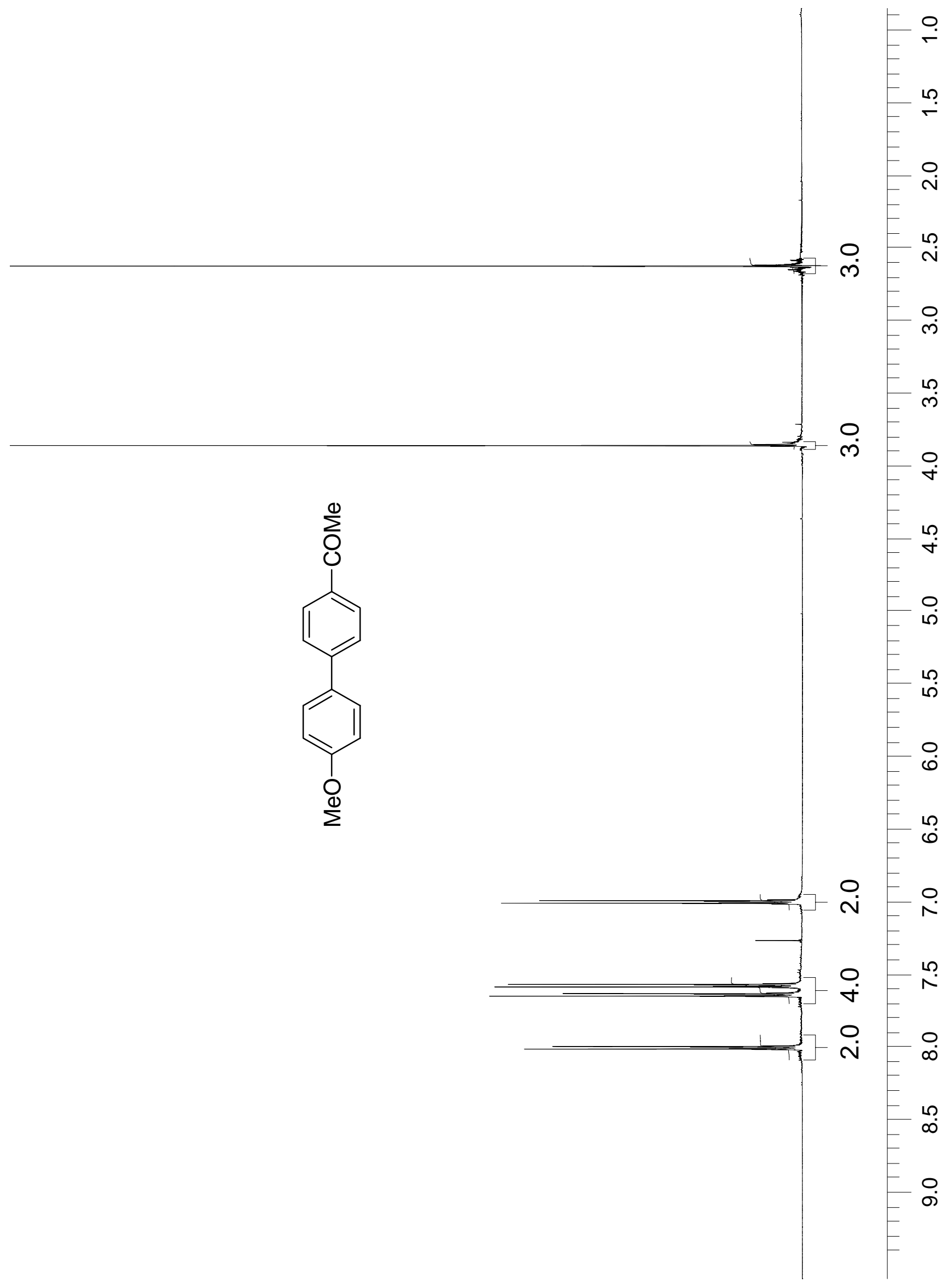

